\title{
Independence, Relative Randomness, and PA Degrees
}

\author{
Adam R. Day and Jan Reimann
}

\begin{abstract}
We study pairs of reals that are mutually Martin-Löf random with respect to a common, not necessarily computable probability measure. We show that a generalized version of van Lambalgen's theorem holds for noncomputable probability measures, too. We study, for a given real $A$, the independence spectrum of $A$, the set of all $B$ such that there exists a probability measure $\mu$ so that $\mu\{A, B\}=0$ and $(A, B)$ is $(\mu \times \mu)$-random. We prove that if $A$ is computably enumerable (c.e.), then no $\Delta_{2}^{0}$-set is in the independence spectrum of $A$. We obtain applications of this fact to PA degrees. In particular, we show that if $A$ is c.e. and $P$ is of PA degree so that $P \nsucceq_{\mathrm{T}} A$, then $A \oplus P \geq_{\mathrm{T}} \emptyset^{\prime}$.
\end{abstract}

\section{Independence and Relative Randomness}

The property of independence is central to probability theory. Given a probability space with measure $\mu$, we call two measurable sets $\mathcal{A}$ and $\mathscr{B}$ independent if

$$
\mu \mathscr{A}=\frac{\mu(\mathcal{A} \cap \mathscr{B})}{\mu \mathscr{B}} .
$$

The idea behind this definition is that if event $\mathcal{B}$ occurs, it does not make event $\mathcal{A}$ any more or less likely. This paper considers a similar notion, that of relative randomness. The theory of algorithmic randomness provides a means of defining which elements of Cantor space $\left(2^{\omega}\right)$ are random. We call $A \in 2^{\omega}$ Martin-Löf random if $A$ is not an element of any effective null set. We denote the class of all Martin-Löf random reals by MLR.

We say that $A$ is Martin-Löf random relative to $B$, or $A \in \operatorname{MLR}(B)$ if $A$ is not an element of any null set effective in $B$. Relative randomness is analogous to independence because if $A \in \operatorname{MLR}(B)$, then not only is $A$ a random real but even given the information in $B$, we cannot capture $A$ in an effective null set. If we start

Received July 10, 2012; accepted August 10, 2012

2010 Mathematics Subject Classification: Primary 03D32

Keywords: algorithmic randomness, independence, PA degrees, c.e. sets, van Lambalgen's theorem

(C) 2014 by University of Notre Dame 10.1215/00294527-2377842 
with the assumption that $A$ and $B$ are both Martin-Löf random, then the following theorem of van Lambalgen establishes that relative randomness is symmetrical.

Theorem 1.1 (van Lambalgen [12]) If $A, B \in \operatorname{MLR}$, then $A \in \operatorname{MLR}(B)$ if and only if $B \in \operatorname{MLR}(A)$ if and only if $A \oplus B \in \operatorname{MLR}$.

We can extend the notion of relative randomness to any probability measure. We take $\mathcal{P}\left(2^{\omega}\right)$ to be the set of all Borel probability measures on Cantor space. Endowed with the weak-* topology, $\mathcal{P}\left(2^{\omega}\right)$ becomes a compact metrizable space. The measures that are a finite, rational-valued, linear combination of Dirac measures form a countable dense subset, and one can choose a metric on $\mathcal{P}\left(2^{\omega}\right)$ that is compatible with the weak-* topology so that the distance between those basic measures is a computable function, and with respect to which $\mathcal{P}\left(2^{\omega}\right)$ is complete. In other words, $\mathcal{P}\left(2^{\omega}\right)$ can be given the structure of an effective Polish space. We can represent measures via Cauchy sequences of basic measures. This allows for coding measures as reals, and one can show that there exists a continuous, surjective mapping $\rho: 2^{\omega} \rightarrow \mathcal{P}\left(2^{\omega}\right)$ such that for any $X \in 2^{\omega}$,

$$
\rho^{-1}(\{\rho(X)\}) \text { is a } \Pi_{1}^{0}(X) \text {-class. }
$$

For details of this argument, see Day and Miller [2]. If $\mu \in \mathcal{P}\left(2^{\omega}\right)$, any real $R$ with $\rho(R)=\mu$ is called a representation of $\mu$.

We want to define randomness relative to a parameter with respect to a probability measure $\mu$. Martin-Löf's framework easily generalizes to tests that have access to an oracle. However, our test should have access to two sources: the parameter of relative randomness and the measure (in the form of a representation).

Definition 1.2 Let $R_{\mu}$ be a representation of a measure $\mu$, and let $A \in 2^{\omega}$.

1. An $\left(R_{\mu}, A\right)$-test is given by a sequence $\left(\mathcal{V}_{n}: n \in \mathbb{N}\right)$ of uniformly $\Sigma_{1}^{0}\left(R_{\mu} \oplus A\right)$ classes $\mathcal{V}_{n} \subseteq 2^{\omega}$ such that for all $n, \mu\left(\mathcal{V}_{n}\right) \leq 2^{-n}$.

2. A real $X \in 2^{\omega}$ passes an $\left(R_{\mu}, A\right)$-test $\left(\mathcal{V}_{n}\right)$ if $X \notin \bigcap_{n} \mathcal{V}_{n}$.

3. A real $X \in 2^{\omega}$ is $\left(R_{\mu}, A\right)$-random, or $R_{\mu}$-random relative to $A$, if it passes all $\left(R_{\mu}, A\right)$-tests.

If, in the previous definition, $A=\emptyset$, we simply speak of an $R_{\mu}$-test and of $X$ being $R_{\mu}$-random.

The previous definition defines randomness with respect to a specific representation. If $X$ is random for one representation, it is not necessarily random for other representations. On the other hand, we can ask whether a real exhibits randomness with respect to some representation, so the following definition makes sense.

Definition 1.3 A real $X \in 2^{\omega}$ is $\mu$-random relative to $A \in 2^{\omega}$, or simply $\mu$ - $A$ random, if there exists a representation $R_{\mu}$ of $\mu$ such that $X$ is $\left(R_{\mu}, A\right)$-random. We denote by $\operatorname{MLR}_{\mu}(A)$ the set of all $\mu$ - $A$-random reals.

For Lebesgue measure $\lambda$, we sometimes suppress the measure. Hence, in accordance with established notation, $\operatorname{MLR}(A)$ denotes the set of all Martin-Löf random reals.

A most useful property of the theory of Martin-Löf randomness is the existence of universal tests. Universal tests subsume all other tests. Furthermore, they can be defined uniformly with respect to any parameter. The construction can be extended to tests with respect to a measure $\mu$. More precisely, there exists a uniformly c.e. 
sequence $\left(U_{n}: n \in \mathbb{N}\right)$ of sets $U_{n} \subseteq 2^{<\omega}$ such that if we set, for $R, A \in 2^{\omega}$,

$$
U_{n}^{R, A}=\left\{[\sigma]:\left\langle\sigma, \tau_{0}, \tau_{1}\right\rangle \in U_{n}, \tau_{0} \prec R, \tau_{1} \prec A\right\},
$$

then $\left(U_{n}^{R, A}\right)$ is an $(R, A)$-test and $X \in 2^{\omega}$ is $(R, A)$-random if and only if $X \notin \bigcap_{n} U_{n}^{R, A}$. We call $\left(U_{n}\right)$ a universal oracle test.

Since for any $R \in 2^{\omega}, \rho^{-1}(\rho(R))$ is $\Pi_{1}^{0}(R)$, we can eliminate the representation of a measure in a test for randomness by defining, for any $A \in 2^{\omega}$,

$$
\widetilde{U}_{n}^{R, A}=\bigcap_{S \in \rho^{-1}(\{\rho(R)\})} u_{n}^{S, A} .
$$

The resulting class $\widetilde{U}_{n}^{A}$ is still $\Sigma_{1}^{0}(R)$, since $\rho^{-1}(\{\rho(R)\})$ is $\Pi_{1}^{0}(R)$ and hence compact.

Proposition 1.4 For any $R, A \in 2^{\omega}$ with $\rho(R)=\mu$, a real $X$ is $\mu$-A-random if and only if

$$
X \notin \bigcap_{n} \widetilde{u}_{n}^{R, A} .
$$

Proof If $X$ is $\mu$-A-random, then it passes every $\left(R_{\mu}, A\right)$-test for some representation $R_{\mu}$ of $\mu$, in particular, the instance $\left(U_{n}^{R_{\mu}, A}\right)$ of the universal oracle test. Since $R_{\mu} \in \rho^{-1}(\{\rho(R)\})$, it follows that $X$ passes $\widetilde{U}_{n}^{R, A}$.

On the other hand, if for every representation $R_{\mu}$ of $\mu, X$ fails the test $\left(U_{n}^{R, A}\right)$, then $X \in \bigcap_{n} \widetilde{U}_{n}^{R, A}$.

Proposition 1.4 shows that the test $\widetilde{U}_{n}^{R, A}$ is related to the concept of a uniform test, originally introduced by Levin [8], and further developed by Gács [4] and Hoyrup and Rojas [5]. Hence we call it a uniform oracle test. Note that if $R, S$ are both representations of a measure $\mu$, then the uniform oracle tests $\left(\widetilde{U}_{n}^{R, A}\right)_{n}$ and $\left(\widetilde{U}_{n}^{S, A}\right)_{n}$ are identical.

Definition 1.5 Take $A, B \in 2^{\omega}$ and $\mu \in \mathcal{P}\left(2^{\omega}\right)$. We say that $A$ and $B$ are relatively random with respect to $\mu$ if $A \in \operatorname{MLR}_{\mu}(B)$ and $B \in \operatorname{MLR}_{\mu}(A)$.

Note that the representations of $\mu$ witnessing randomness for $A$ and $B$, respectively, do not have to be identical. If $A$ and $B$ are relatively random with respect to some measure $\mu$, then $\mu$ might offer some information about the relationship between $A$ and $B$. For example, we know that if $A$ and $B$ are relatively random with respect to Lebesgue measure, then any real they both compute must be K-trivial.

A trivial case of relative randomness occurs when $A$ and $B$ are both atoms of the underlying measure. A real $X$ is an atom of a measure $\mu$ if $\mu\{X\}>0$. A measure with no atoms is called nonatomic. Given this, perhaps the most obvious question to ask about relative randomness is the following.

Question 1.6 For which $A, B \in 2^{\omega}$ does there exist a measure $\mu$ such that $A$ and $B$ are relatively random with respect to $\mu$ and neither $A$ nor $B$ is an atom of $\mu$ ?

This question is closely related to a theorem of Reimann and Slaman [10]. They proved that an element $X$ of Cantor space is noncomputable if and only if there exists a measure $\mu$ such that $X$ is $\mu$-random and $X$ is not an atom of $\mu$.

Van Lambalgen's theorem shows that $A$ and $B$ are relatively random if and only if $A \oplus B \in$ MLR. If we take $\lambda$ to be the uniform measure, then $A \oplus B \in$ MLR 
if and only if the pair $(A, B) \in 2^{\omega} \times 2^{\omega}$ is Martin-Löf random with respect to the product measure $\lambda \times \lambda$, that is, $(A, B) \in \operatorname{MLR}_{\lambda \times \lambda}$. We begin our investigation into relative randomness by showing that van Lambalgen's theorem holds for any Borel probability measure on Cantor space.

Theorem 1.7 Let $\mu \in \mathcal{P}\left(2^{\omega}\right)$, and let $A, B \in 2^{\omega}$. Then $(A, B) \in \operatorname{MLR}_{\mu \times \mu}$ if and only if $A \in \operatorname{MLR}_{\mu}$ and $B \in \operatorname{MLR}_{\mu}(A)$, that is, if and only if $A$ and $B$ are relatively random with respect to $\mu$.

Proof Let $R$ be any representation of $\mu$. First, let us consider if $B \notin \operatorname{MLR}_{\mu}(A)$. In this case we have that $B \in \bigcap_{n} U_{n}^{R, A}$. We define an $(R, \emptyset)$-test for $2^{\omega} \times 2^{\omega}$ by $\mathcal{V}_{n}^{R}=\left\{[\tau] \times[\sigma]: \exists \eta \prec R\left(\langle\sigma, \eta, \tau\rangle \in U_{n}\right)\right\}$. This ensures that $(A, B) \in \bigcap_{n} \mathcal{V}_{n}^{R}$. By applying Fubini's theorem we can establish that

$$
\begin{aligned}
(\mu \times \mu)\left(\mathcal{V}_{n}^{R}\right) & =\int_{2^{\omega} \times 2^{\omega}} \chi_{\mathcal{V}_{n}^{R}}(X, Y) d \mu \times d \mu \\
& =\int_{2^{\omega}}\left(\int_{2^{\omega}} \chi_{u_{n}^{R, X}}(Y) d \mu(Y)\right) d \mu(X) \\
& \leq \int_{2^{\omega}} 2^{-n} d \mu(X)=2^{-n} .
\end{aligned}
$$

Hence $(A, B)$ is not $(R, \emptyset)$-random. As this is true for any representation $R$ of $\mu$, we have $(A, B) \notin \operatorname{MLR}_{\mu \times \mu}$. The same argument shows a fortiori that if $A \notin \operatorname{MLR}_{\mu}$, then $(A, B) \notin \operatorname{MLR}_{\mu \times \mu}$.

To establish the other direction, assume that $(A, B) \notin \operatorname{MLR}_{\mu \times \mu}$. Again let $R$ be any representation of $\mu$.

Hence $(A, B) \in \bigcap_{n} \mathcal{V}_{n}^{R}$, where $\left(\mathcal{V}_{n}^{R}\right)$ is a universal $R$-test for $2^{\omega} \times 2^{\omega}$. Let

$$
W_{n}^{R, X}=\left\{Y:(X, Y) \in \mathcal{V}_{n}^{R}\right\} .
$$

We have that $\mathcal{W}_{n}^{R, X}$ is a $\Sigma_{1}^{0}(R \oplus X)$-class and this is uniform in $n$. However, given any $X$, we do not know whether or not $\mu\left(\mathcal{W}_{n}^{R, X}\right) \leq f(n)$ for some decreasing computable function $f$ such that $\lim _{n} f(n)=0$. Hence we cannot necessarily turn this into a Martin-Löf test relative to $X$. In fact, it is not even necessarily true that $\liminf _{n} \mu\left(\mathcal{W}_{n}^{R, X}\right)=0$. We will show that the failure to turn this into a Martin-Löf test for some $X \in 2^{\omega}$ implies that $X \notin \operatorname{MLR}_{\mu}$. This is a slight strengthening of the result that van Lambalgen obtained in his thesis. Van Lambalgen showed that if $\liminf _{n} \mu\left(\mathcal{W}_{n}^{R, X}\right) \neq 0$, then $X \notin \operatorname{MLR}_{\mu}$.

However, we can generalize the proof of van Lambalgen's theorem given in Nies [9]. We define another $R$-test by letting

$$
\mathcal{T}_{n}^{R}=\left\{X \in 2^{\omega}: \mu\left(\mathcal{W}_{2 n}^{R, X}\right)>2^{-n}\right\} .
$$

To see that $\mathcal{T}_{n}^{R} \leq 2^{-n}$, note that

$$
\begin{aligned}
(\mu \times \mu) \mathcal{V}_{2 n}^{R} & \geq \int_{\mathcal{T}_{n}^{R} \times 2^{\omega}} \chi_{\mathcal{V}_{2 n}^{R}}(X, Y) d \mu \times d \mu \\
& =\int_{\mathcal{T}_{n}^{R}} \int_{2^{\omega}} \chi_{\mathcal{W}_{2 n}^{R, X}}(Y) d \mu(Y) d \mu(X) \\
& \geq \int_{\mathcal{T}_{n}^{R}} 2^{-n} d \mu(X)=2^{-n} \mu\left(\mathcal{T}_{n}^{R}\right) .
\end{aligned}
$$


Now as $2^{-2 n} \geq(\mu \times \mu) \mathcal{V}_{2 n}^{R}$, we have $\mu\left(\mathcal{T}_{n}{ }^{R}\right) \leq 2^{-n}$. Hence $\left(\mathcal{T}_{n}^{R}\right)$ is an $R$-test. Assume that $A$ is not $R$-random. Then $A$ avoids all but finitely many of the sets $\mathcal{T}_{n}^{R}$. Hence for all but finitely many $n$ we have $\mu \mathcal{W}_{2 n}^{R, A} \leq 2^{-n}$, and so by modifying finitely many $\mathcal{W}_{2 n}^{R, A}$ we can obtain an $(R, A)$-test that covers $B$. Therefore $B$ is not $R$-random relative to $A$.

For all representations $R$ of $\mu$, we have shown that either $A$ is not $R$-random or $B$ is not $R$-random relative to $A$. However, to prove the theorem, it is essential that we get the same outcome for all representations; that is, if $(A, B) \notin \operatorname{MLR}_{\mu}$, then either for all representations $R$ of $\mu, A$ is not $R$-random or for all representations $R$ of $\mu$, $B$ is not $R$-random relative to $A$.

We can resolve this problem by taking our test $\left(\mathcal{V}_{n}^{R}\right)$ on the product space to be a uniform oracle test. (A uniform oracle test on $2^{\omega} \times 2^{\omega}$ can be defined analogously to the uniform oracle test on $2^{\omega}$ defined above.) In this case we always obtain the same "projection tests" $\left(W_{n}^{R, X}\right)$ (independent of $R$ ) and hence the same outcome for any representation of $\mu$.

Corollary 1.8 If $A \geq_{\mathrm{T}} B$ and $(A, B) \in \mathrm{MLR}_{\mu \times \mu}$, then $B$ must be an atom of $\mu$.

Proof This holds because $B \in \operatorname{MLR}_{\mu}(A)$ if and only if $B$ is an atom of $\mu$.

We note that we cannot extend one direction of van Lambalgen's theorem to product measures of the form $\mu \times v$. In particular, it is not true that if $A \in \mathrm{MLR}_{\mu}$ and $B \in \operatorname{MLR}_{v}(A)$, then $(A, B) \in \operatorname{MLR}_{\mu \times v}$. For example, it is possible to code a real $B$ into a measure $\mu$ in such a way that $B$ is computable from every representation of $\mu$ and choose a measure $v$ so that there exist $A \in \operatorname{MLR}_{\mu}, B \in \operatorname{MLR}_{v}(A)$, with $\mu\{A\}=v\{B\}=0$. But for any such $\mu, v$ we cannot have $(A, B) \in \operatorname{MLR}_{\mu \times v}$, since any $\mu \times \nu$-test has access to $\mu$ and hence can compute $B$.

Given any $X \in 2^{\omega}$, we will use $\mathcal{R}(X)$ to denote the set of reals $Y$ such that $X$ and $Y$ are relatively random with respect to some measure $\mu$ and neither $X$ nor $Y$ are atoms of $\mu$; that is,

$\mathcal{R}(A)=\left\{B \in 2^{\omega}:\left(\exists \mu \in \mathcal{P}\left(2^{\omega}\right)\right)\left[(A, B) \in \operatorname{MLR}_{\mu \times \mu}\right.\right.$, and $\left.\left.\mu\{A\}=\mu\{B\}=0\right]\right\}$.

We call $\mathcal{R}(A)$ the independence spectrum of $A$.

The following proposition lists some basic properties of the independence spectrum.

Proposition 1.9 For all $A, B \in 2^{\omega}$ the following hold:

(1) $A \in \mathcal{R}(B)$ if and only if $B \in \mathcal{R}(A)$;

(2) $B \in \mathcal{R}(A)$ implies that $\left.A\right|_{\mathrm{T}} B$;

(3) if $A$ is noncomputable and $v$ is a nonatomic measure with a computable representation, then $\mathcal{R}(A)$ has $v$-measure 1 ;

(4) if $A \in \operatorname{MLR}$, then $\operatorname{MLR}(A) \subsetneq \mathcal{R}(A)$.

Proof Condition (1) is by definition, and (2) is by Corollary 1.8.

For (3) suppose that $A$ is noncomputable and $v$ is a computable measure with $\nu\{A\}=0$. There is a measure $\mu$ such that $A$ is not an atom of $\mu$ and $A \in \operatorname{MLR}_{\mu}$, say, via a representation $R_{\mu}$. Let $\kappa=(\mu+v) / 2$. There exists a representation $R_{\kappa} \leq_{\mathrm{T}} R_{\mu}$, as $v$ is computable. We claim that $A$ is $R_{\kappa}$-random. For if not, then $A$ fails some $R_{\kappa}$-test $\left(\mathcal{W}_{n}^{R_{\kappa}}\right)$. We have

$$
\mu \mathcal{W}_{n}^{R_{\kappa}}=2 \kappa \mathcal{W}_{n}^{R_{\kappa}}-v \mathcal{W}_{n}^{R_{\kappa}} \leq 2 \kappa \mathcal{W}_{n}^{R_{\kappa}} \leq 2^{n-1} .
$$


Since $R_{\kappa} \leq_{\mathrm{T}} R_{\mu},\left(W_{n+1}^{R_{\kappa}}\right)$ would define an $R_{\mu}$-test that covers $A$, contradicting the assumption that $A$ is $R_{\mu}$-random. Furthermore, by assumption on $\mu$ and $\nu$, $\kappa\{A\}=0$. Hence

$$
\left.\operatorname{MLR}_{\kappa}(A) \backslash\{B: \kappa\{B\} \neq 0\}\right) \subseteq \mathcal{R}(A)
$$

by van Lambalgen's theorem.

Now $v\left(\operatorname{MLR}_{\kappa}(A)\right)=1$ because the complement of $\operatorname{MLR}_{v}(A)$ is a $\kappa$-null set and hence a $v$-null set ( $v$ is absolutely continuous with respect to $\kappa$ by definition). Moreover, the set of atoms of $\kappa$ is countable and so has $\nu$-measure 0 by the assumption that $v$ is nonatomic. This gives us

$$
v\left(\operatorname{MLR}_{\kappa}(A) \backslash\{B: \kappa\{B\} \neq 0\}\right)=1
$$

and thus $\nu \mathcal{R}(A)=1$.

For (4) suppose that $A$ is Martin-Löf random. By the definition of $\mathcal{R}(A)$ and Theorem 1.7 we have $\operatorname{MLR}(A) \subseteq \mathcal{R}(A)$.

On the other hand, $A$ is not computable, and hence by (3), $\mathcal{R}(A)$ has measure 1 for any computable, nonatomic measure. Let $v$ be a computable, nonatomic measure orthogonal to Lebesgue measure (e.g., the (1/3,2/3)-Bernoulli measure). Since $\nu \mathcal{R}(A)=1, \mathcal{R}(A)$ has to contain a $v$-random element $X$. But $X$ cannot be relatively Martin-Löf random. Therefore, $\operatorname{MLR}(A) \subsetneq \mathcal{R}(A)$.

The proposition shows that, outside the upper and lower cone of a real $A$, the complement of $\mathcal{R}(A)$ is rather small measurewise. On the other hand, the above properties leave open the possibility that $\mathcal{R}(A)$ is just the set of reals that are Turing incomparable with $A$. We will now establish that this is not necessarily the case.

Proposition 1.10 Let $R$ be a representation of a measure $\mu$. If $A \in 2^{\omega}$ is such that

1. A is c.e.,

2. $A$ is $R$-random, and

3. $A$ is not an atom of $\mu$,

then $R \oplus A \geq_{\mathrm{T}} R^{\prime}$.

Proof Given such an $R$ and $A$, let $A_{s}$ be a computable approximation to $A$. We define the function $f \leq_{\mathrm{T}} A \oplus R$ by

$$
f(x)=\min \left\{s:(\exists m \leq s)\left(A_{s} \uparrow m=A \uparrow m \wedge \mu_{s}[A \uparrow m]<2^{-x}\right)\right\} .
$$

In this definition we take $\mu_{s}[\sigma]$ to be an $R$-computable approximation to $\mu[\sigma]$ from above. Note that $f$ is well defined because $A$ is not an atom of $\mu$. We claim that if $g$ is any partial function computable in $R$, then for all but finitely many $x \in \operatorname{dom}(g)$, we have $f(x)>g(x)$. To establish this claim, let $g$ be an $R$-computable partial function. We will build an $R$-test $\left\{U_{n}\right\}_{n \in \omega}$ by defining $U_{n}$ to be

$$
\left\{X \in 2^{\omega}:(\exists x>n)(\exists m)\left(g(x) \downarrow \wedge \mu\left[A_{g(x)} \uparrow m\right]<2^{-x} \wedge X \succ\left(A_{g(x)} \uparrow m\right)\right)\right\} .
$$

Because any $x \in \operatorname{dom}(g)$ adds a single open set $\left(\left[A_{g(x)} \uparrow m\right]\right.$ for some $\left.m\right)$ of measure less than $2^{-x}$ to those $U_{n}$ with $n<x$, we have constructed a valid test. Now if $g(x) \downarrow \geq f(x)$, then by definition of $f$, there is some $m \leq f(x)$ such that $\mu[A \uparrow m]<2^{-x}$ and $A \uparrow m=A_{f(x)} \uparrow m=A_{g(x)} \uparrow m$. Here we use the fact that $A$ is c.e. Thus for all $n<x, A \in U_{n}$. Because $A$ is $R$-random, we have $f(x)>g(x)$ for all but finitely many $x$ in $\operatorname{dom}(g)$. 
Let $g(x)$ be the $R$-computable partial function with domain $R^{\prime}$ such that $g(x)$ is the unique $s$ such that $x \in R_{s+1}^{\prime} \backslash R_{s}^{\prime}$. For almost all $x$, we have $x \in R^{\prime}$ if and only if $x \in R_{f(x)}^{\prime}$, and so $R^{\prime} \leq_{\mathrm{T}} A \oplus R$.

Theorem 1.11 Let $R$ be a representation of a measure $\mu$. If

1. A is c.e.,

2. A is $\mu$-random, and

3. $A$ is not an atom of $\mu$,

then $R \oplus A \geq_{\mathrm{T}} \emptyset^{\prime}$.

Proof Note the following characteristics of the previous proof. First, the totality of $f$ does not depend on the fact that $A$ is $R$-random; it only depends on the fact that $A$ is not an atom of $\mu$. The construction is uniform, so there is a single index $e$ such that $\Phi_{e}(A \oplus \hat{R})$ is total if $\hat{R}$ is any representation of $\mu$. Additionally, if $A$ is $\hat{R}$-random, then for all but finitely many $x, \Phi_{e}(A \oplus \hat{R} ; x) \geq g(x)$, where $g$ is any $\hat{R}$-computable partial computable function.

Let $R$ be any representation of $\mu$. The set $\{A \oplus \hat{R}: \hat{R}$ is a representation of $\mu\}$ is a $\Pi_{1}^{0}(A \oplus R)$-class, and $\Phi_{e}$ is total on this class. From $A \oplus R$ we can compute a function $f$ that dominates $\Phi_{e}(A \oplus \hat{R})$, where $A$ is $\hat{R}$-random. As $f$ dominates any $\hat{R}$-computable partial function, we have $A \oplus R \geq_{\mathrm{T}} \emptyset^{\prime}$.

Corollary 1.12 If $A$ is c.e. and $B \leq_{\mathrm{T}} \emptyset^{\prime}$, then $B \notin \mathcal{R}(A)$.

The question remains, however, how big the independence spectrum of a real can be outside its upper and lower cones.

Question 1.13 Is the set of all $X$ such that $\left.X\right|_{\mathrm{T}} A$ and $X \notin \mathcal{R}(A)$ countable?

\section{Computably Enumerable Sets and PA Degrees}

We will now give two (somehow unexpected) applications of Theorem 1.11 to the interaction between c.e. sets and sets of PA degree. Recall that a set $A \subseteq \mathbb{N}$ is of $P A$ degree if it is Turing equivalent to a set coding a complete extension of Peano arithmetic (PA). PA degrees have many interesting computability-theoretic properties. For instance, a set is of PA degree if and only if it computes a path through every nonempty $\Pi_{1}^{0}$-class. However, a complete degree-theoretic characterization of the PA degrees is still not known. If $A \geq_{\mathrm{T}} \emptyset^{\prime}$, then $A$ is of PA degree. On the other hand, Gödel's first incompleteness theorem implies that no c.e. set can be a complete extension of PA. Jockusch and Soare [6] showed, moreover, that if a set is of incomplete c.e. degree, it cannot be of PA degree. This also follows from the Arslanov completeness criterion, since any PA degree computes a fixed-point free function (see [1]).

It seems therefore worthwhile to gain a complete understanding how c.e. sets and PA degrees are related. The crucial fact that links Theorem 1.11 to PA degrees is a result by Day and Miller [2]. They showed that every set of PA degree computes a representation of a neutral measure. Such a measure has the property that every real is random with respect to it, that is, $2^{\omega}=\operatorname{MLR}_{\mu}$. The existence of neutral measures was first established by Levin [8].

Our first result shows that below $\emptyset^{\prime}$, c.e. sets and PA degrees behave quite complementary. 
Corollary 2.1 (to Theorem 1.11) If $A$ is a c.e. set and $P$ a set of PA degree such that $P \geq_{\mathrm{T}} A$, then $P \oplus A \geq_{\mathrm{T}} \emptyset^{\prime}$.

Proof By the result of Day and Miller [2] mentioned above, $P$ computes a representation $R_{\mu}$ of a neutral measure $\mu$ and $A \in \operatorname{MLR}_{\mu}$. Day and Miller [2] also showed that a real $X$ is an atom of a neutral measure if every representation of the measure computes $X$. Now because $P \Varangle_{\mathrm{T}} A$, we have that $A$ is not an atom of $\mu$. Thus all hypotheses of Theorem 1.11 are satisfied, and we have $P \oplus A \geq_{\mathrm{T}} \emptyset^{\prime}$.

Corollary 2.1 strengthens a result due to Kučera and Slaman (unpublished). Recall that a function $f: \mathbb{N} \rightarrow \mathbb{N}$ is diagonally noncomputable if $f(n) \neq \varphi_{n}(n)$ for all $n$, where $\varphi_{n}$ denotes, as usual, the $n$th partial computable function. Kučera and Slaman constructed a low 2 c.e. set so that $A \oplus f \equiv_{\mathrm{T}} \emptyset^{\prime}$ for any diagonally noncomputable function $f \leq_{\mathrm{T}} \emptyset^{\prime}$. It is well known that every PA degree computes a $\{0,1\}$-valued diagonally noncomputable function. Hence the set constructed by Kučera and Slaman joins any PA degree below $\emptyset^{\prime}$ to $\emptyset^{\prime}$. As we will see below (Corollary 2.4), Corollary 2.1 yields that this is in fact true for any nonlow c.e. set.

One can ask which kind of incomplete c.e. sets can be bounded by PA degrees below $\emptyset^{\prime}$. This question was first raised by Kučera [7].

For which incomplete c.e. sets $A$ does there exist a set $P$ of $P A$ degree such that $A<_{\mathrm{T}} P<_{\mathrm{T}} \emptyset^{\prime}$ ?

We can use Corollary 2.1 to completely answer this question. We say that a set $B$ is of $P A$ degree relative to a set $A$, written $B \gg A$ (see Simpson [11]), if $B$ computes a path through every nonempty $\Pi_{1}^{0}(A)$-class. One well-known fact we will make use of is the following. If $P$ is of $P A$ degree, then there exists a set $Q$ of $P A$ degree such that $P \gg Q$. One way to prove this fact is to observe that the $\Pi_{1}^{0}$-class

$$
\left\{(A, B) \in 2^{\omega} \times 2^{\omega}: A \in \mathrm{DNR}_{2} \wedge B \in \mathrm{DNR}_{2}(A)\right\}
$$

is nonempty, where $\mathrm{DNR}_{2}$ and $\mathrm{DNR}_{2}(A)$ are the classes of $\{0,1\}$-valued diagonally noncomputable functions and $\{0,1\}$-valued diagonally noncomputable functions relative to $A$, respectively.

Theorem 2.2 If $A$ is a c.e. set, then the following are equivalent.

(1) $A$ is low.

(2) There exist $P, P \gg A$, and $P$ is low.

(3) There exists $P$ of $P A$ degree such that $\emptyset^{\prime}>_{\mathrm{T}} P>_{\mathrm{T}} A$.

Proof $\quad(1) \Rightarrow(2)$ : There is a (nonempty) $\Pi_{1}^{0}(A)$-class of sets $B \gg A$. Relativize the low basis theorem to find $P \gg A$ and $P^{\prime} \equiv_{\mathrm{T}} A^{\prime}$. As $A$ is low, so is $P$.

(2) $\Rightarrow$ (3): This is clear.

(3) $\Rightarrow$ (1): Take any $Q$ of PA degree such that $P \gg Q$. Now $Q \geq_{\mathrm{T}} A$ because otherwise $Q \oplus A \geq \emptyset^{\prime}$, but this is impossible because $P \geq_{\mathrm{T}} Q \oplus A$ and $P \geq_{\mathrm{T}} \emptyset^{\prime}$. Hence $P \gg A$. But now we have that $\emptyset^{\prime}$ is c.e. in $A$ and also that $\emptyset^{\prime}$ computes a DNR function relative to $A$. Hence by relativizing Arslanov's completeness criterion we have $A^{\prime} \equiv_{\mathrm{T}} \emptyset^{\prime}$.

Observe that in the proof of $(3) \Rightarrow(1)$, showing $P \gg A$ only used the facts that $P \Varangle_{T} \emptyset^{\prime}$ and $P \geq_{T} A$. Hence we get the following corollary.

Corollary 2.3 If $P$ is a set of $P A$ degree and $A$ is a c.e. set such that $P \geq_{T} A$ and $P \nsucceq_{T} \emptyset^{\prime}$, then $P \gg A$.

We also obtain the strengthening of the Kučera-Slaman result mentioned above. 
Corollary 2.4 If $A$ is a nonlow c.e. set, then $A \oplus P \equiv_{\mathrm{T}} \emptyset^{\prime}$ for all $P \leq_{\mathrm{T}} \emptyset^{\prime}$ of $P A$ degree.

\section{Notes}

1. For a comprehensive presentation of the theory of Martin-Löf randomness, see the monographs by Downey and Hirschfeldt [3] and Nies [9].

2. We mean here, of course, that $A$ is computably enumerable viewed as a subset of $\mathbb{N}$, by identifying a subset of $\mathbb{N}$ with the real given by its characteristic sequence.

3. After the authors announced the result presented in Corollary 2.1, proofs not involving measure-theoretic arguments have been found independently by A. Kučera and J. Miller.

\section{References}

[1] Arslanov, M. M., "On some generalizations of the fixed-point theorem," Soviet Mathematics, vol. 25 (1981), pp. 1-10.

[2] Day, A. R., and J. S. Miller, "Randomness for non-computable measures," Transactions of the American Mathematical Society, vol. 365 (2013), pp. 3575-91. MR 3042595. DOI 10.1090/S0002-9947-2013-05682-6. 2, 7, 8

[3] Downey, R. G., and D. R. Hirschfeldt, Algorithmic Randomness and Complexity, Theory and Applications of Computability, Springer, New York, 2010. MR 2732288. DOI 10.1007/978-0-387-68441-3. 9

[4] Gács, P., "Uniform test of algorithmic randomness over a general space," Theoretical Computer Science, vol. 341 (2005), pp. 91-137. Zbl 1077.68038. MR 2159646. DOI 10.1016/j.tcs.2005.03.054.

[5] Hoyrup, M., and C. Rojas, "Computability of probability measures and Martin-Löf randomness over metric spaces," Information and Computation, vol. 207 (2009), pp. 83047. Zbl 1167.68023. MR 2519075. DOI 10.1016/j.ic.2008.12.009.

[6] Jockusch, C. G., Jr., and R. I. Soare "Degrees of members of $\Pi_{1}^{0}$ classes," Pacific Journal of Mathematics, vol. 40 (1972), pp. 605-16. Zbl 0209.02201. MR 0309722. 7

[7] Kučera, A., "Remarks on randomness and PA sets," conference presentation at "Logic, Computability, and Randomness," Cordoba, Argentina, 2004.

[8] Levin, L. A., "Uniform tests for randomness," Doklady Akademii Nauk SSSR, vol. 227 (1976), pp. 33-35. MR 0414222. 3, 7

[9] Nies. A., Computability and Randomness, vol. 51 of Oxford Logic Guides, Oxford University Press, Oxford, 2009. MR 2548883. DOI 10.1093/acprof:oso/9780199230761 $.001 .0001 .4,9$

[10] Reimann, J., and T. A. Slaman, "Measures and their random reals," preprint, arXiv:0802.2705v2 [math.LO].

[11] Simpson, S. G., "Degrees of unsolvability: A survey of results," pp. 631-52 in Handbook of Mathematical Logic, edited by J. Barwise, vol. 90 of Studies in Logic and the Foundations of Mathematics, North-Holland, Amsterdam, 1977. MR 0457132.

[12] M. van Lambalgen. "Random sequences," Ph.D. dissertation, University of Amsterdam, Amsterdam, 1987.

\section{Acknowledgments}

Reimann was partially supported by National Science Foundation grant DMS-1201263. The authors would like to thank Steve Simpson for stimulating and insightful discussions. 
Day

School of Mathematics, Statistics and Operations Research Victoria University of Wellington

Wellington 6140

New Zealand

adam.day@vuw.ac.nz

Reimann

Department of Mathematics

Pennsylvania State University

University Park

State College, Pennsylvania 16802

USA

reimann@math.psu.edu 